But if conservation is to include the possibility of continuing evolution then the minimum population size is 500 . Once this conclusion is reached the problems of conservation become apparent. The minimum territory of a pair of mountain lions is $52 \mathrm{~km}^{2}$; to conserve this species requires an area about twice the size of Yellowstone National Park. There are therefore few reserves in the world that are large enough to ensure the survival of the dominant carnivores, and there is no doubt the majority are doomed to disappear.

Yet nature reserves present the only possible solution. In which case there are criteria of population genetics and biology which must be observed if reserves are to be designed to function properly. Size is crucial according to island biogeographical theory, but so is migration. As a result there are arguments that small reserves are possible if there are connecting corridors. But the authors point out that this will only work in a limited number of cases.

Even the $r$ selected species, notably our crop plants and their wild relatives, have problems. It is crucial that we maintain genetic diversity to satisfy our present and future needs for food production. This means genes to cope with specific individual problems such as day length or disease, and genes by which we can build up co-adapted complexes for characters such as yield. The authors show that these genes are scattered in wild and cultivated material, often in places that cannot be predicted.

For conservation and continued evolution, a diversity of populations must be maintained, if possible in natural conditions. So far, because of geographical expansion from migration and trade, the diversity in these species has actually increased. But now the success of modern agriculture and plant breeding has sent the process into reverse, and whole groups of cultivars, such as the Welsh upland wheats and barleys, have completely disappeared. The solutions endorsed by the authors are various: in situ and ex situ dynamic conservation, and static conservation by storage, of which seed storage is perhaps the most efficient. But they do not offer any easy solutions to the problem of what to store, except to emphasize the need for international co-operation.

This book is a remarkable survey of a problem which is crucial to us all. Not only does it list over 600 references but it achieves a remarkable synthesis and perspective, based on a masterly understanding of theory as well as practice. It is enormously readable because of its simple and enthusiastic style. It is critical reading for plant and animal breeders, and all ecologists, so that they can better argue with politicians about what has to be done, before it is too late.

A.D. Bradshaw is Professor of Botany at the University of Liverpool.

\title{
Liquid crystal measurement
}

\section{J. W. Emsley}

Physical Properties of Liquid Crystalline Materials. By W.H. De Jeu. Pp.133. ISBN 0-677-04040-7. (Gordon and Breach: 1980.) $\$ 35.25$.

EVERY schoolboy knows that there are liquid crystal display devices, but a relatively few graduates are familiar with this "fourth state of matter". There is certainly a need for introductory texts on the physical properties of these materials, to supplement George Gray's excellent book, Molecular Structure and the Properties of Liquid Crystals (Academic, 1962), which covers their general properties. This short book by De Jeu is the first of what promises to be a series, edited by Professor Gray, on liquid crystals. It has, however, a misleading title since it does not cover all physical properties and is confined to discussing only the simplest mesophase, the nematic. This does have the advantage of making it a book that will be read rather than one too complex and long to appeal to those interested in other aspects of liquid crystals.

Five physical properties are discussed: magnetic susceptibility, refractive index, dielectric permittivity, elastic constants and viscosity coefficients. Each topic is introduced by a discussion of the basic physics, which makes heavy demands on the background knowledge of the reader.
There follows a survey of methods of measurement and a discussion of the work which has been done. The author draws heavily on his own research and does not attempt a general review, but this again adds to the readability of the book and conveys a sense that much remains to be done.

Physicists, chemists and engineers interested in liquid crystallinity will welcome this book as providing a short, readable account of the five physical properties. It is written primarily with experimentalists in mind and it has a particularly useful chapter on sample preparation. It is less successful at explaining the significance of the results, mainly because of a deliberate decision not to get involved with the details of the various theoretical models developed in recent years. Restricting the scope of the book to those topics in which the author has been directly involved was a wise decision, for although one might wish for more discussion of cholesteric or smectic phases, or a more critical review of theory, what is included is discussed with an authority derived from a long experience in thelaboratory.

J. W. Emsley is a Senior Lecturer in the Department of Chemistry at the University of Southampton.

\section{Consolidating fungal biochemistry}

\section{P.G. Mantle}

Lipid Biochemistry of Fungi and Other Organisms. By J. D. Weete with contributions by D.J. Weber. Pp.388. ISBN 0-306-40570-9. (Plenum: 1981.) \$45, £28.35.

THIS volume is a reincarnation of the authors' previous book, Fungal Lipid Biochemistry, published by Plenum in 1974. The text has been completely rewritten and revised but still centres on the distribution and biochemistry of fungal lipids - fatty acids, acylglycerols, sterols, phospholipids, aliphatic hydrocarbons and sphingolipids. New aspects covered in the present volume include a brief history of fungal lipid research, more comprehensive treatments of lipid taxonomy and substrate conversion into stored lipid, a description of the multifunctional yeast enzyme for fatty acid synthesis, the biosynthesis of polyprenols and carotenoids, and a review of the role of fungal lipids in sporulation and spore germination. The bibliography has been correspondingly increased and, where appropriate, reference is made to contrasting bacterial, plant and animal systems.

A few minor typographical errors can be recognized and I was somewhat surprised to find myself credited (Table 3.7) with research in the field of phycomycete fatty acids. Nevertheless, the authors should be congratulated on condensing the essence of about 1,000 research papers into a lucid text supplemented liberally by comprehensive tables and figures.

It is often only when someone gives the time and care to review a subject that the gaps in our knowledge of an otherwise diffuse topic become well defined. Fungal lipid metabolism is a case in point. Thirty years ago this book would have been simply a pamphlet listing some of the oils contained in fungi. Biochemistry has since moved so fast that the lipid composition of representatives of the major groups of fungi is now fairly well documented and the biosynthetic routes are generally understood. It is, however, notable that although regulatory mechanisms for fatty acid synthesis have been well researched, the regulatory interactions involving lipids in whole-organism metabolism are largely unknown. This is perhaps not surprising since it is bound to be very complex. Nevertheless, it is such areas of knowledge which 